\title{
Aplidin (plitidepsin) is a novel anti-myeloma agent with potent anti-resorptive activity mediated by direct effects on osteoclasts
}

\author{
Jesus Delgado-Calle ${ }^{1,2,4}$, Noriyoshi Kurihara ${ }^{1}$, Emily G. Atkinson ${ }^{2}$, Jessica Nelson ${ }^{1}$, \\ Kazuaki Miyagawa ${ }^{1}$, Carlos Maria Galmarini ${ }^{5}$, G. David Roodman ${ }^{1,4,6}$ and Teresita \\ Bellido $2,3,4,6$ \\ ${ }^{1}$ Department of Medicine, Division of Hematology/Oncology, Indiana University School of Medicine, Indianapolis, IN, USA \\ ${ }^{2}$ Department of Anatomy and Cell Biology, Indiana University Sc hool of Medicine, Indianapolis, IN, USA \\ ${ }^{3}$ Department of Medicine, Division of Endocrinology, Indiana University School of Medicine, Indianapolis, IN, USA \\ ${ }^{4}$ Indiana Center for Musculoskeletal Health, Indiana University School of Medicine, Indianapolis, IN, USA \\ ${ }^{5}$ Research Department, PharmaMar S.A., Madrid, Spain \\ ${ }^{6}$ Roudebush VA Medical Center, Indianapolis, IN, USA \\ Correspondence to: Jesus Delgado-Calle, email: jedelgad@iupui.edu \\ Teresita Bellido, email: tbellido@iupui.edu
}

Keywords: myeloma; osteoclasts; osteocytes; osteoblasts; tumor

Received: November 26, $2018 \quad$ Accepted: March 23, 2019

Published: April 12, 2019

Copyright: Delgado-Calle et al. This is an open-access article distributed under the terms of the Creative Commons Attribution License 3.0 (CC BY 3.0), which permits unrestricted use, distribution, and reproduction in any medium, provided the original author and source are credited.

\section{ABSTRACT}

Despite recent progress in its treatment, Multiple Myeloma (MM) remains incurable and its associated bone disease persists even after complete remission. Thus, identification of new therapeutic agents that simultaneously suppress MM growth and protect bone is an unmet need. Herein, we examined the effects of Aplidin, a novel anti-cancer marine-derived compound, on MM and bone cells. In vitro, Aplidin potently inhibited MM cell growth and induced apoptosis, effects that were enhanced by dexamethasone (Dex) and bortezomib (Btz). Aplidin modestly reduced osteocyte/osteoblast viability and decreased osteoblast mineralization, effects that were enhanced by Dex and partially prevented by Btz. Further, Aplidin markedly decreased osteoclast precursor numbers and differentiation, and reduced mature osteoclast number and resorption activity. Moreover, Aplidin reduced Dex-induced osteoclast differentiation and further decreased osteoclast number when combined with Btz. Lastly, Aplidin alone, or suboptimal doses of Aplidin combined with Dex or Btz, decreased tumor growth and bone resorption in ex vivo bone organ cultures that reproduce the 3D-organization and the cellular diversity of the $\mathrm{MM} /$ bone marrow niche. These results demonstrate that Aplidin has potent anti-myeloma and antiresorptive properties, and enhances proteasome inhibitors blockade of MM growth and bone destruction.

\section{INTRODUCTION}

Multiple myeloma (MM) is the second most common hematologic malignancy and the most frequent cancer that involves bone $[1,2]$. The bone/bone marrow (BM) niche plays a critical role in $\mathrm{MM}$ onset and progression. MM cells locate in specialized niches in the $\mathrm{BM}$ where they interact with stromal cells, endothelial cells, immune cells, osteoblasts, osteoclasts, adipocytes, and osteocytes [2, 3]. These interactions transform the bone/BM niche into an ideal environment for the proliferation and survival of MM cells. Further, actively expanding MM cells increase osteoclast numbers and activity, leading to development of lytic bone lesions that rarely heal due to a concomitant suppression of osteoblast bone forming activity $[1,4]$. MM can cause pathologic 
fractures and excruciating bone pain, which increase morbidity and mortality and diminish the quality of life of patients. Remarkable progress has been made in the treatment of $\mathrm{MM}$ in the past 10 years, however MM remains incurable in the majority of patients. In addition, osteolytic lesions persist in patients in remission with no evidence of marrow infiltration by MM cells [4, 5], thus prompting a continued search for additional therapeutic options that inhibit MM growth and protect bone health.

Aplidin is a novel anti-cancer compound isolated from the marine tunicate Aplidium albicans [6-8]. In vitro studies showed that Aplidin has anti-MM activity against 19 MM cell lines including cells resistant to anti-MM agents frequently used in the clinic (i.e. melphalan, doxorubicin, thalidomide derivatives, and dexamethasone) and primary MM cells isolated from patients (13 out 16 showed response to Aplidin) [9]. Recently, Losada et al demonstrated that Aplidin targets the eukaryotic elongation factor 1A2 (EF1A2), which is overexpressed in MM cells [7]. Mechanistically, several pathways have been identified to mediate the effects of Aplidin on the viability of MM cells. Aplidin induces apoptosis in MM cells, which involves activation of p38 and c-jun $\mathrm{NH}(2)$-terminal kinase signaling, Fas/CD95 translocation to lipid rafts, and ultimately caspase activation. In addition, Aplidin decreases the proliferation of MM cells, an effect mediated by the suppression of several proliferative genes. [9, 10]. In vivo, it has been reported that Aplidin has anti-MM activity in preclinical animal models [9, 10]. Further, in a pivotal phase III trial of patients with relapsed or refractory MM, Aplidin in combination with dexamethasone (Dex) significantly reduced the risk of progression or death compared to Dex alone [11]. However, the effects of Aplidin, alone or in combination with anti-MM therapy, on bone cells and MM-induced bone disease are unknown.

In this study, we examined the effects of Aplidin, alone or in combination with other anti-MM drugs, on MM cells and bone cells (osteocytes, osteoblasts, and osteoclasts). Using in vitro approaches and an ex vivo 3D model of MM bone disease, we found that Aplidin decreased MM cell viability, and that this action was enhanced by the anti-MM drugs Dex and Bortezomib (Btz). In addition, Aplidin modestly decreased osteocyte and osteoblast viability, and this effect was exacerbated by Dex, but partially prevented by Btz. Importantly, Aplidin potently inhibited osteoclast precursor commitment and differentiation, inhibited mature osteoclast bone resorption, and reduced Dex-induced increases in osteoclast differentiation. These findings demonstrate that Aplidin inhibits both tumor growth and bone resorption, and suggest that Aplidin can enhance the clinical efficacy of proteasome inhibitors by potentiating their anti-tumor properties and reducing the risk of skeletal-related events by inhibiting resorption through acting on osteoclasts.

\section{RESULTS}

\section{The anti-myeloma effects of Aplidin are enhanced by dexamethasone and bortezomib}

We first determined the dose- and time-dependent effects of Aplidin on the viability of murine and human MM cell lines. Concentrations higher than $1 \mathrm{nM}$ of Aplidin decreased the viability of human JJN3 MM cells in a dose-dependent manner (EC50 10 nM) and progressively reduced MM cell viability from $24 \mathrm{~h}$ to $48 \mathrm{~h}$ (Figure 1A and 1C). Aplidin also decreased the viability of murine 5TGM1 MM cells (Figure 1B). Aplidin induced MM cell death in a dose and time dependent manner in both JJN3 and 5TGM1 MM cells (Figure $1 \mathrm{~A}$ and $1 \mathrm{~B}$ ), with an $\mathrm{EC}_{50}$ of $\sim 10 \mathrm{nM}$ Aplidin for JJN3 MM cells and $20 \mathrm{nM}$ for 5TGM1 cells after $48 \mathrm{~h}$ of treatment (Figure 1C), and decreased the proliferation of JJN3 MM cells (Figure 1D). The elevated MM cell death induced by Aplidin was due to apoptosis, as treatment with the caspase 3 inhibitor DEVD fully prevented Aplidin-induced increases in MM cell death (Figure 1D). In contrast, DEVD did not affect the number of alive MM cells, which remained decreased by Aplidin (Figure 1D).

We next evaluated the effects of combinations of Aplidin with other anti-MM drugs on MM cell viability/ cell death. Treatment with Dex alone decreased the viability of JJN3 cells, increased MM cell death up to $23 \%$, and enhanced the effect of $10 \mathrm{nM}$ Aplidin on MM cell death by 1.6 -fold (39\% vs $63 \%$ cell death, Aplidin vs Aplidin+Dex, respectively; Figure 1E). Btz also decreased JJN3 viability by $50 \%$, augmented JJN3 cell death up to $35 \%$, and increased the number of JJN3 dead cells in combination with $10 \mathrm{nM}$ Aplidin by 2.5 -fold (20\% vs $50 \%$ cell death, Aplidin vs Aplidin+Btz, respectively; Figure $1 \mathrm{~F})$. These results demonstrate that Aplidin induces MM cell death and its anti-MM activity is further enhanced when combined with Dex and Btz.

\section{Aplidin-mediated decrease in osteocyte viability is enhanced by dexamethasone and partially prevented by bortezomib}

To gain insight into the effects of Aplidin in the skeleton, we first investigated its effects on osteocytes, the most abundant cells in bone. Aplidin 10nM increased the percentage of dead MLO-A5 osteocyte-like cells up to $10-15 \%$ after $48 \mathrm{~h}$ (Figure $2 \mathrm{~A}$ ), and inhibit MLO-A5 osteocyte-like proliferation (Figure 2A, 2B). Mechanistic studies showed that Aplidin increases osteocyte cell death by inducing apoptosis. As shown in Figure 2B, treatment with the caspase 3 inhibitor DEVD fully blocked Aplidininduced increases in osteocyte cell death; however, treatment with DEVD did not prevent the decreased in the number of alive osteocytes induced by Aplidin. Further, 
as shown previously [12], treatment with Dex increased osteocyte cell death to $\sim 8 \%$, and in combination with Aplidin $10 \mathrm{nM}$, Dex further increased Aplidin-induced osteocyte cell death by 1.8 -fold (19\% vs $37 \%$ cell death, Aplidin vs Aplidin+Dex, respectively; Figure 2C). In contrast, Btz did not alter osteocyte cell death and partially blunted the increase in dead osteocytes induced by Aplidin $10 \mathrm{nM}$ (31\% vs 19\%, Aplidin vs Aplidin+Btz, respectively) (Figure 2D).
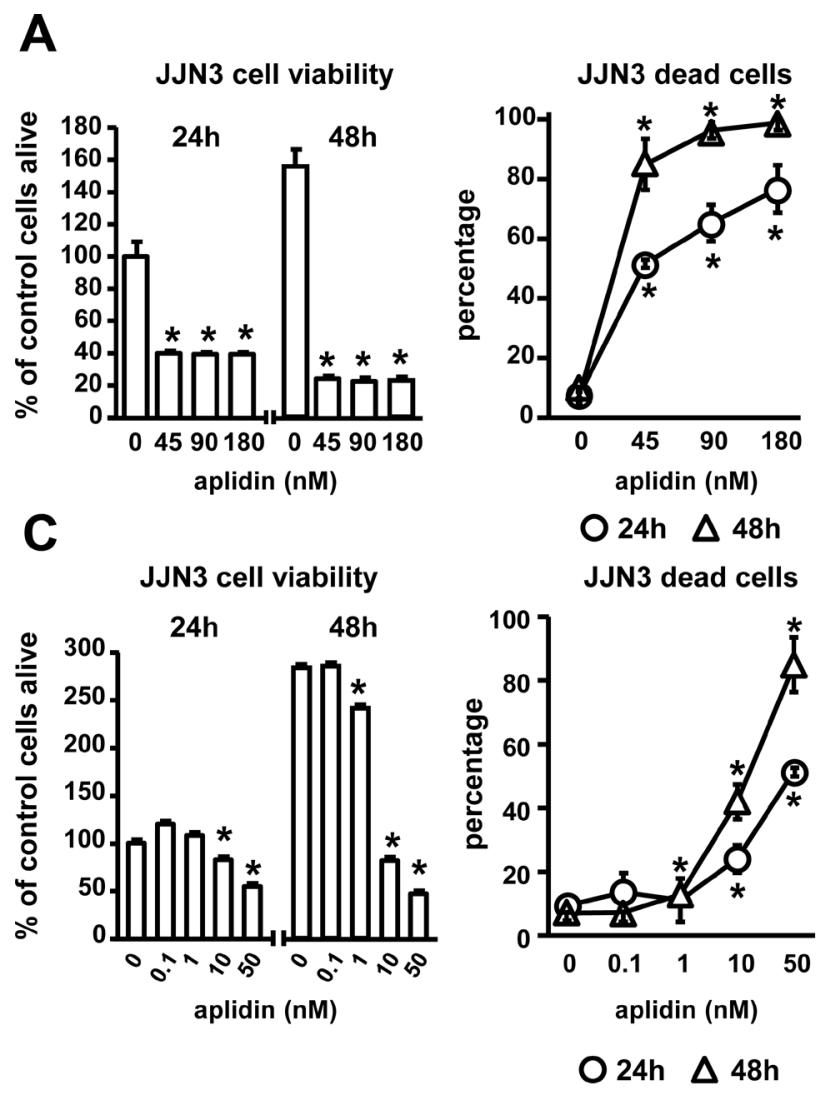

E JJN3 cell viability (48h)

JJN3 dead cells (48h)
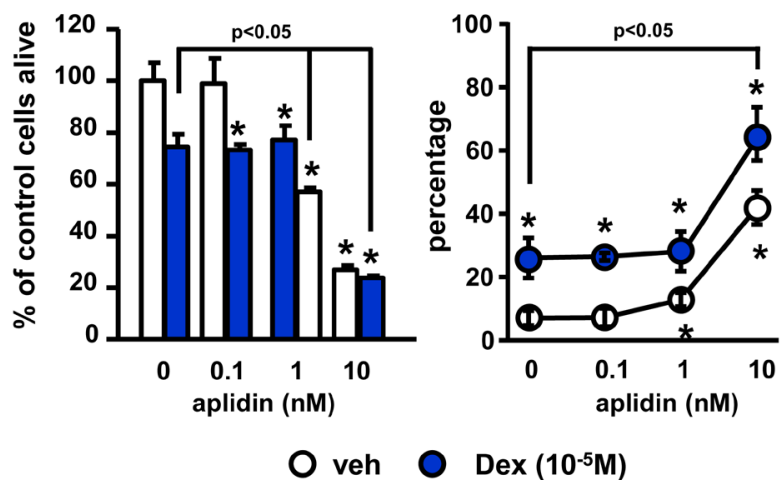

Co-administration of bortezomib partially prevents the decreases in osteoblast viability and function induced by Aplidin

We next examined the actions of Aplidin on osteoblasts. Concentrations of $1 \mathrm{nM}$ Aplidin and higher increased cell death up to $10-20 \%$ after $48 \mathrm{~h}$ in OB-6 osteoblastic cells (Figure 3A). As previously demonstrated, Dex increased osteoblast cell death, and when combined with
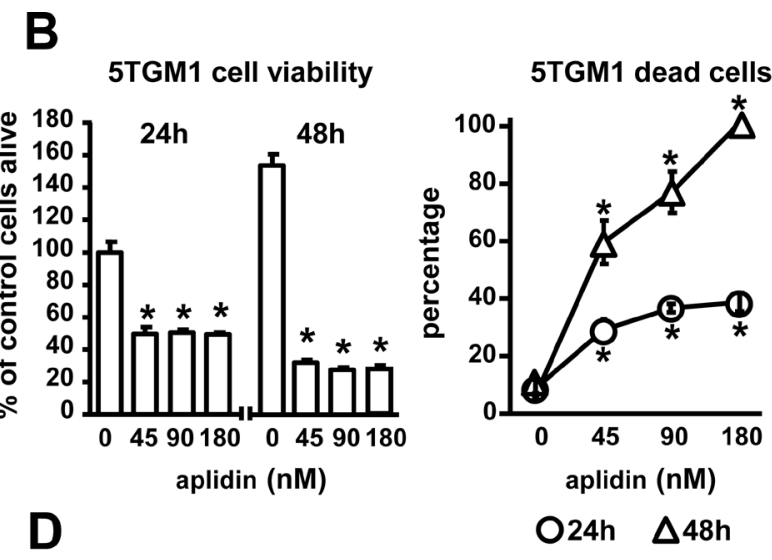

JJN3 alive cells (48h)

JJN3 dead cells (48h)
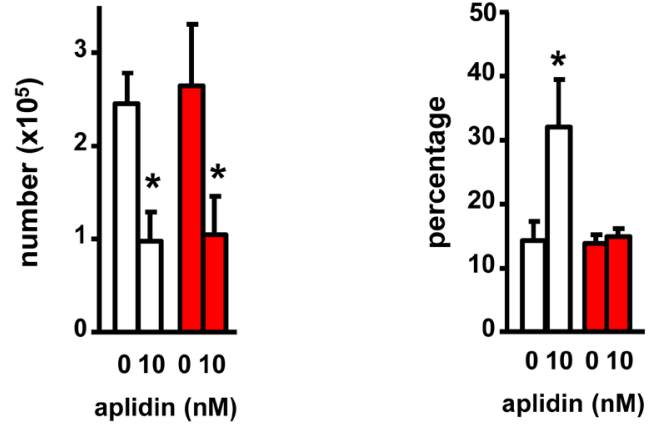

$\mathbf{F}$

veh DEVD (100nM)

JJN3 cell viability (48h)

JJN3 dead cells (48h)
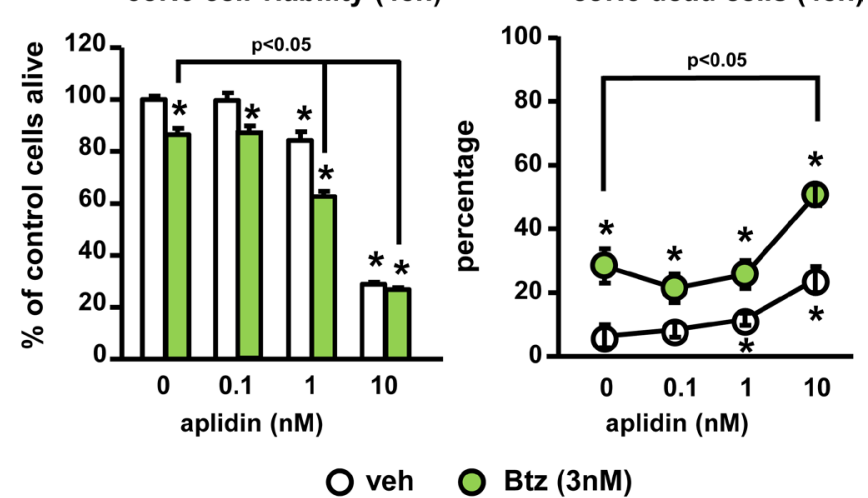

Figure 1: The inhibition of MM cell viability by Aplidin is enhanced by Dex and Btz. (A-C) Human JJN3 and murine 5TGM1 MM cells were treated with increasing concentrations of Aplidin and MM cell viability/death was evaluated after $24 \mathrm{~h}$ and $48 \mathrm{~h}$ using MTT and Trypan blue uptake assays. JJN3 MM cells were treated with Aplidin $10 \mathrm{nM}$ with/without DEVD (D), and increasing concentrations of Aplidin in the presence/absence of a fixed dose of Dex (E) or Btz (F) and cell viability/death was evaluated after $48 \mathrm{~h}$. Representative experiments out of two are shown $\left(n=4-6\right.$ per condition). Bars represent means \pm SD. ${ }^{*} p<0.05$ vs vehicle; lines indicate $p<0.05$ for Dex/Btz alone vs Dex/Btz + Aplidin. 
Aplidin 1 and $10 \mathrm{nM}$, it further enhanced this effect (10\% vs 15\%, Aplidin $10 \mathrm{nM}$ vs Aplidin $10 \mathrm{nM}+$ Dex, respectively) (Figure 3B). As observed in osteocytes cultures, Btz did not alter the viability of osteoblasts; however, it partially reduced the increases in the number of dead osteoblasts induced by $10 \mathrm{nM}$ Aplidin (8\% vs 3\%, Aplidin $10 \mathrm{nM}$ vs Aplidin $10 \mathrm{nM}+$ Dex, respectively) (Figure $3 \mathrm{C}$ ).

Continuous (5 days a week) and pulse (once a week) administration of 1-10 $\mathrm{nM}$ Aplidin decreased the matrix production and mineralization of OB-6 cells cultured under osteogenic conditions by $20 \%$ (Figure $3 \mathrm{D}$ and $3 \mathrm{E})$. Further, consistent with the protective effects of Btz against Aplidin-induced osteoblast cell death, co-administration of Btz partially prevented ( $50 \%)$ the reduction in mineralization induced by Aplidin 1 $\mathrm{nM}$ (Figure 3E). Similar to the findings in OB-6 cells, continuous treatment with Aplidin $10 \mathrm{nM}$ decreased matrix mineralization in pre-osteoblastic MC4 cells (Figure 3F). This effect was associated with decreases in the mRNA expression of Runx2, Collagen 1a, and alkaline phosphatase (Figure 3F).

\section{Aplidin inhibits osteoclast precursor differentiation and the bone resorbing activity of mature osteoclasts}

We also investigated the effects of Aplidin on osteoclast formation and mature osteoclasts. In vitro,
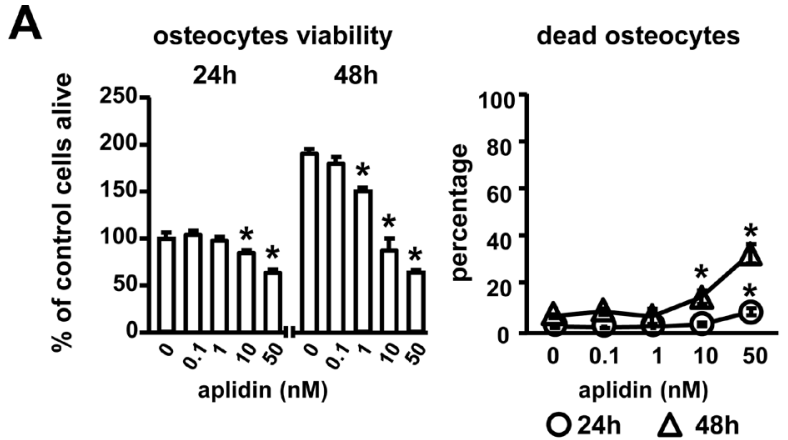

C

osteocyte viability $(48 \mathrm{~h})$
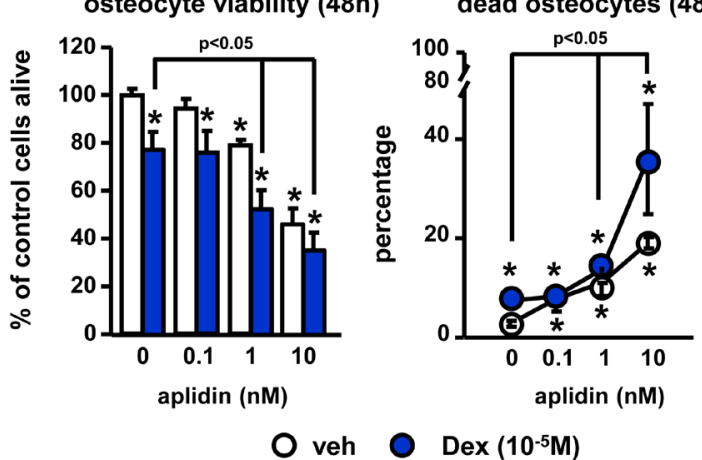

osteoclast lineage cells exhibited higher sensitivity to Aplidin as compared to MM cells or cells of the osteoblastic lineage, as $\mathrm{nM}$ concentrations of Aplidin fully blocked the formation of CFU-GM colonies and osteoclast formation. Thus, the drug was tested at lower concentrations. $50 \mathrm{pM}$ Aplidin decreased by $15 \%$ the number of CFU-GM colonies in cultures of murine bone marrow cells (Figure 4A). Moreover, concentrations as low as $0.01 \mathrm{pM}$ of Aplidin reduced TRAP positive mononuclear osteoclast precursors by $10 \%$ (Figure 4B). In addition, Aplidin decreased the number of multinucleated osteoclasts by $40 \%$ (Figure 4C). Aplidin blunted the rapid (5-15 min) increase in ERK phosphorylation induced by RANKL, but did not affect RANKL-induced p38 phosphorylation (Figure 4D), suggesting that Aplidin inhibited RANKL-induced differentiation of osteoclast precursors by preventing ERK activation. Treatment with Aplidin also decreased the RANKL-mediated induction of markers of osteoclast differentiation, including NFATc1, TRAP, and MMP-9 (Figure 4E). Further, treatment with Dex induced a 3.3-fold increase in the number of osteoclasts induced by RANKL, whereas Btz decreased the differentiation of osteoclast precursors by $\sim 50 \%$ compared to vehicle-treated cells (Figure 4F). Addition of Aplidin to Dex treated marrow cultures resulted in a $25 \%$ reduction in the increase of TRAP + multinucleated cells induced by Dex, and further decreased the number of osteoclasts in pre-osteoclast cultures treated with Btz (Figure 4F).
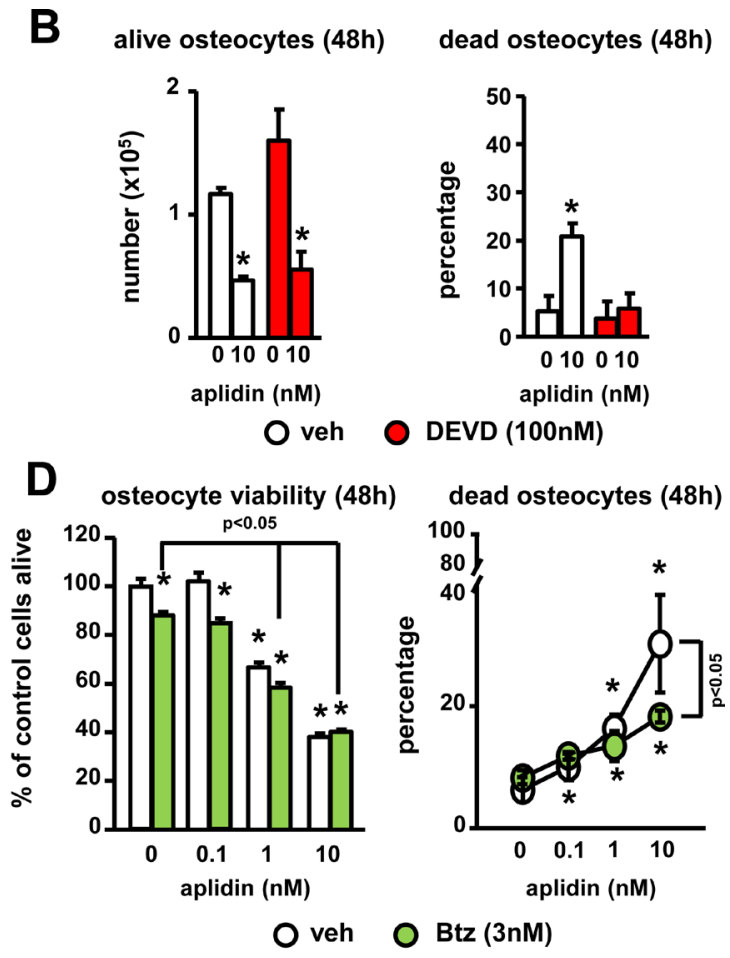

Figure 2: Aplidin decreases osteocyte viability. (A) Time (24-48 h) and dose-response (0-10 nM) to Aplidin in murine MLO-A5 osteocyte-like cells. Evaluation of the effects of combinations of Aplidin with the caspase 3 inhibitor DEVD (B), Dex (C) and Btz (D) after $48 \mathrm{~h}$ treatment. Representative experiments out of two are shown $\left(n=4-6\right.$ per condition). Bars represent means $\pm \mathrm{SD}$. ${ }^{*} p<0.05$ vs vehicle; lines indicate $p<0.05$ for Dex/Btz alone vs Dex/Btz + Aplidin, and Aplidin vs Aplidin + Btz. 
We then tested the effects of Aplidin on mature bone resorbing osteoclasts. Aplidin decreased the number of mature osteoclasts in a dose-dependent manner, and 10
pM Aplidin reduced the increase in Cathepsin $\mathrm{K}$ induced by RANKL in mature osteoclasts (Figure 5A). In addition, Aplidin, either alone and in combination with Dex and
A
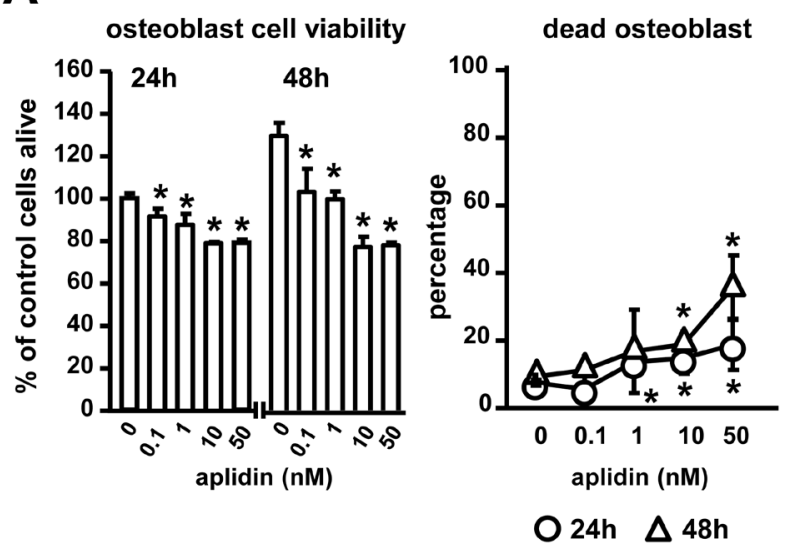

B

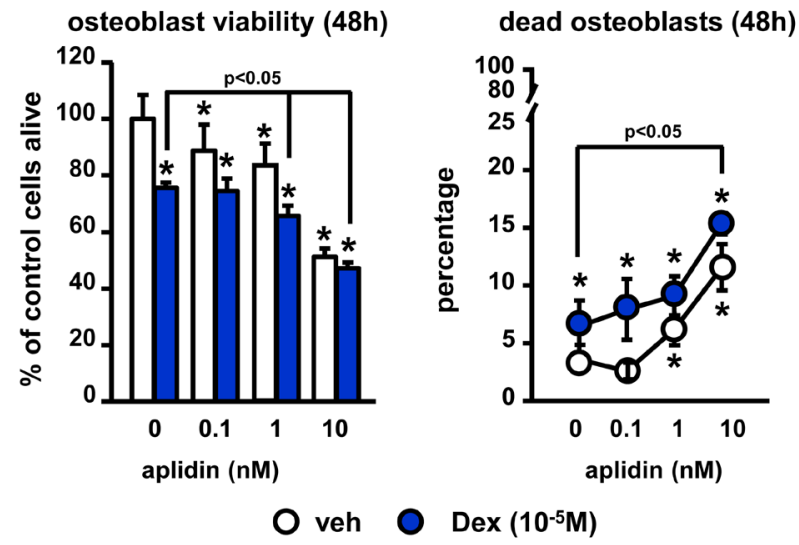

C osteoblast viability (48h)

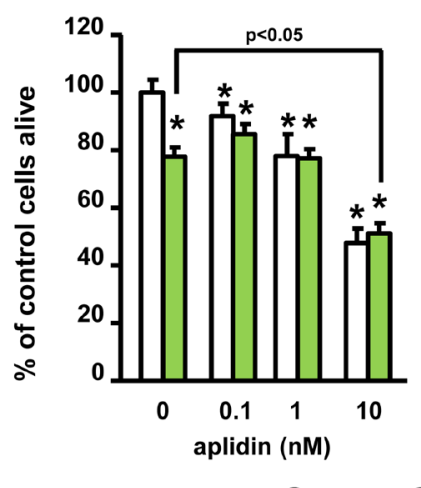

O veh

$\mathrm{E}$

E
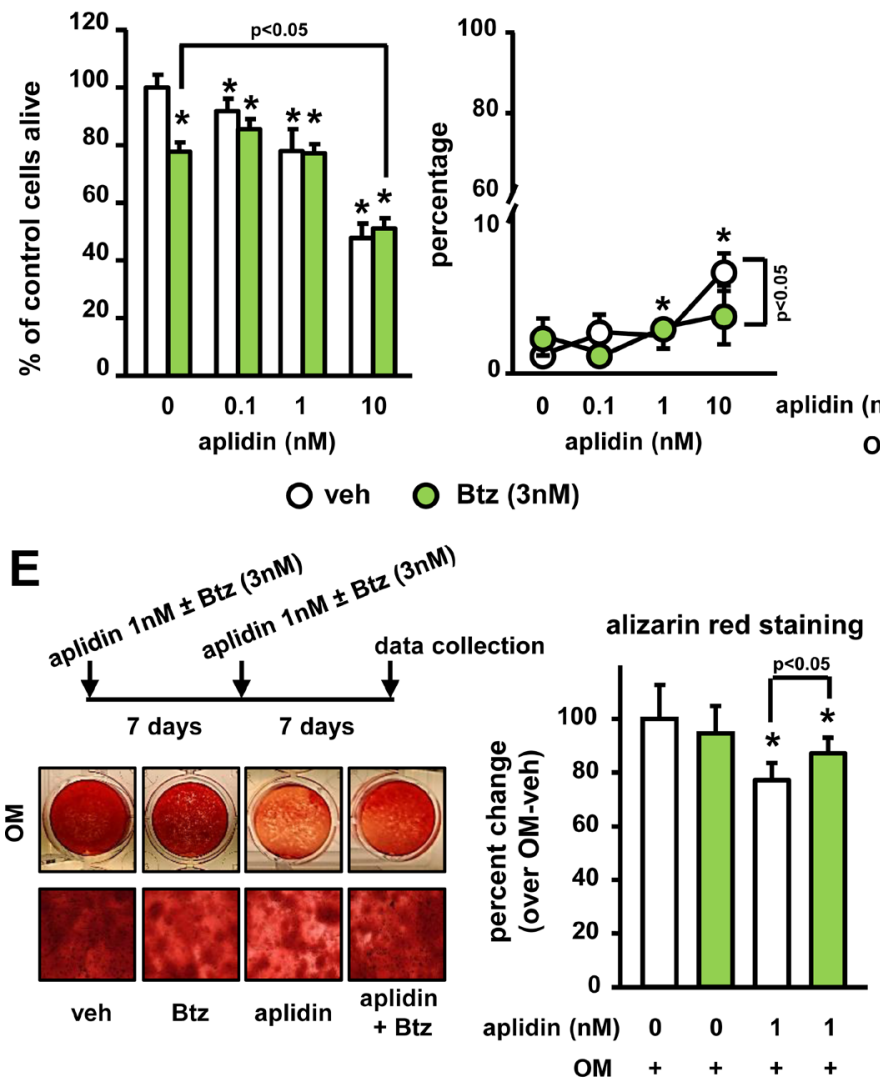

Btz (3nM)

O veh $O \mathrm{Btz}(3 \mathrm{nM})$ aplidin aplidin aplidin aplidin data collection
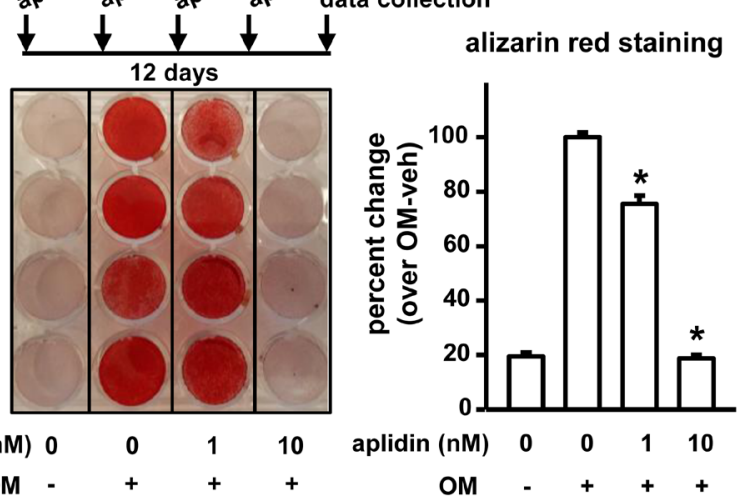

Figure 3: Aplidin decreases osteoblast viability and function and co-administration of Btz partially prevents these effects. (A-C) Cell viability and cell death was evaluated in murine OB-6 osteoblastic cells treated with Aplidin $(0-10 \mathrm{nM})$ alone or in combination with Dex or Btz. Representative experiments out of two are shown ( $n=4-6$ per condition). Mineralization of OB-6 (D-E) and MC-4 (F) cells treated with/without Aplidin in the presence/absence of Btz for 14-21 days evaluated by Alizarin Red staining. Percent change was calculated over cells cultured with osteogenic media $(\mathrm{OM})$ in the absence of Aplidin. Representative experiments out of two are shown $\left(n=3-4\right.$ per condition). Bars represent means \pm SD. ${ }^{*} p<0.05$ vehicle; lines indicate $p$ values $<0.05$ comparing Dex/Btz alone vs Dex/Btz + Aplidin, and Aplidin vs Aplidin + Btz. 
Btz, markedly decreased the number of resorption pits formed by mature osteoclasts plated on bovine bone slices. (Figure 5B and Supplementary Figure 1).

\section{Aplidin exhibits anti-myeloma and anti- resorptive activities in an ex vivo 3D bone organ model of multiple myeloma}

We next examined the effects of Aplidin on tumor growth and bone resorption using an ex vivo bone organ culture model of MM in which the three-dimensional organization and the cellular diversity of the $\mathrm{MM} /$ bone marrow niche are preserved, thus providing a physiologically relevant environment (Figure 6A) [13-16]. After 3 days of culture, conditioned media (CM) collected from calvarial bones bearing MM cells exhibited a 19-fold increase in levels of the tumor marker IgG2b compared to $\mathrm{CM}$ derived from control bones. Three days of treatment with $10 \mathrm{nM}$ Aplidin reduced the levels of IgG2b by $60 \%$ in $\mathrm{CM}$ from bones bearing MM cells (Figure 6B). After 12 days of treatment, Aplidin completely blocked tumor growth, as $\operatorname{IgG} 2 b$ levels in CM from bones bearing MM cells treated with Aplidin were indistinguishable from those found in $\mathrm{CM}$ from control bones (Supplementary Figure 2A). In this particular experiment, we were not able to detect significant increases in the levels of the bone resorption marker collagen type 1 cross-linked C-telopeptide (CTX) in $\mathrm{CM}$ from bones bearing MM. However, treatment with $10 \mathrm{nM}$ Aplidin for 3 days markedly decreased CTX to levels below those found in CM collected from control bones (Figure 6B). Further, CTX levels were undetectable

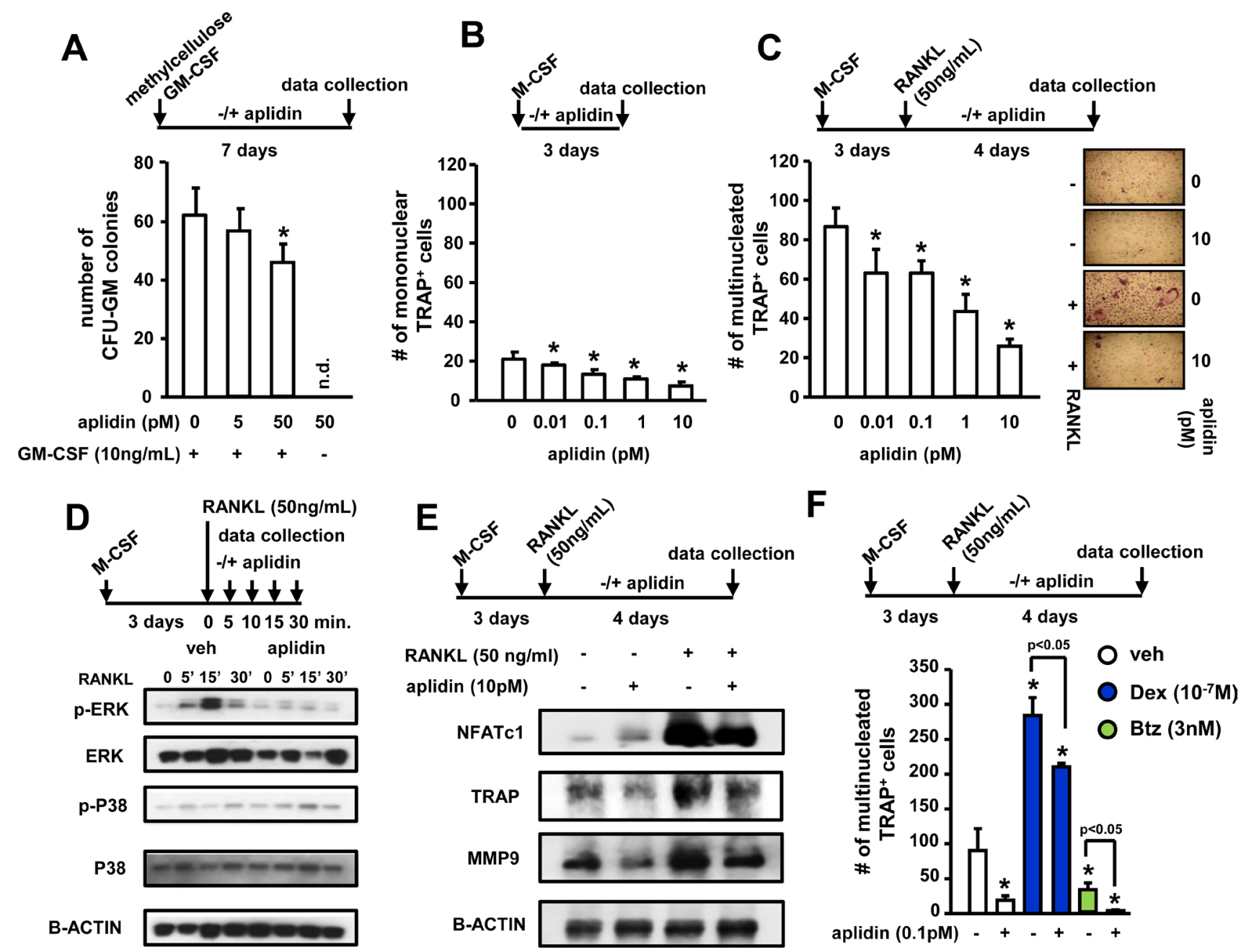

Figure 4: Aplidin inhibits osteoclast precursor expansion and differentiation. (A) Evaluation of the effects of Aplidin (0-50 $\mathrm{pM}$ ) on proliferation and differentiation of hematopoietic progenitors (CFU-GM), (B) expansion of the osteoclast precursor population induced by M-CSF, and (C) RANKL-induced differentiation of osteoclast precursors. Representative images of osteoclast cultures are shown in panel C. (D) Analysis of protein levels of p-ERK, ERK, p-P38, and P38 in cell lysates collected from osteoclast precursors stimulated with RANKL (0-30 minutes). (E) Analysis of the protein levels of NFATc1, TRAP, and MMP9. (F) Number of TRAP+ osteoclasts after treatment with Aplidin in the presence/absence of Dex and Btz. Representative experiments out of two are shown $(n=$ 4 per condition). Bars represent means $\pm \mathrm{SD} .{ }^{*} p<0.05$ vs vehicle; lines indicate $p$ values $<0.05$ comparing Dex/Btz alone vs Dex/Btz + Aplidin. 
in bones treated with 10nM Aplidin for 12 days (Supplementary Figure 2A). These findings are consistent with our in vitro studies showing decreased number of osteoclasts after Aplidin treatment, and support the notion that, in addition to its potent anti-MM properties, Aplidin also has the potential to block bone destruction by inhibiting bone resorption.

We next investigated in this model the effects of combinations of suboptimal doses of Aplidin, Dex, and Btz on tumor growth and bone resorption. Three days of treatment with $1 \mathrm{nM}$ of Aplidin or Btz as single agents did not significantly decrease IgG2b levels in CM from bones bearing MM cells, whereas treatment with Dex alone modestly decreased MM growth. In contrast, combined administration of Aplidin and Dex or Aplidin and Btz significantly decreased tumor burden, demonstrating that Dex and Btz exhibit additive effects with Aplidin, enhancing its anti-MM properties (Figure 6C). After 12 days, $1 \mathrm{nM}$ Aplidin alone decreased tumor growth by $40 \%$, and no additional significant decreases in MM cell burden occurred when combined with Dex or Btz (Supplementary Figure 2B).

$\mathrm{CM}$ from bones bearing $\mathrm{MM}$ and treated under vehicle conditions cells had increased CTX levels compared to control bones not bearing MM cells (Figure 6C). Treatment with Aplidin, Dex, or Btz alone did not alter the increased CTX levels in CM MM myelomabearing bones after 3 days. In contrast, treatment with combinations of Aplidin and Dex decreased CTX levels in CM from MM-bearing bones (Figure 6C). Coadministration of Aplidin and Btz also decreased CTX levels, however these results did not reach statistical

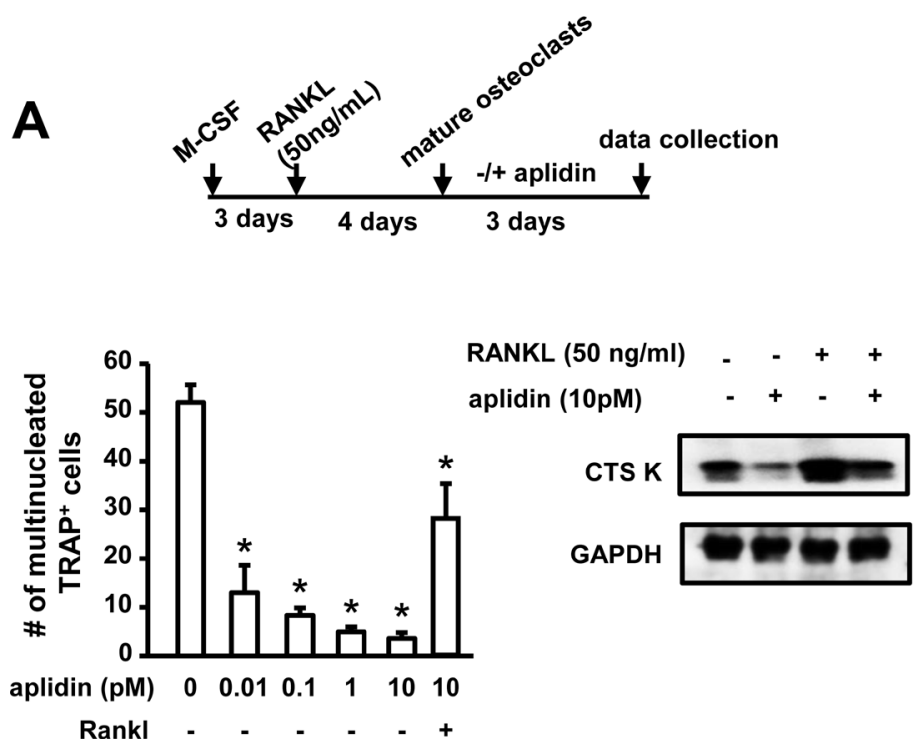

significance (Figure 6C). After 12 days, Aplidin alone reduced CTX in bones bearing MM by $60 \%$. Moreover, Aplidin in combination with Dex and Btz, completely prevented the increased bone resorption induced by MM cells, with CTX levels no different from those observed in $\mathrm{CM}$ from control bones (Supplementary Figure 2B).

\section{DISCUSSION}

Bone destruction is the hallmark of MM, with approximately $70 \%$ MM patients presenting with osteolytic lesions at the time of diagnosis. Despite the abundance of new drugs recently approved for the treatment of MM, the disease remains incurable and the skeletal consequences persist even after achievement of long-term tumor remission. Further, many anti-tumor treatments have adverse effects on bone (i.e. Dex, alkylators, long-term bisphosphonate administration), resulting in more rapid and severe bone destruction [5]. Thus, new more effective therapeutic approaches that improve anti-tumor responses and maintain bone health in patients with $\mathrm{MM}$ are needed. The results of this study demonstrate that Aplidin decreases MM growth and enhances the efficacy of other chemotherapy drugs (Btz and Dex) on MM cells (Figure 7). In addition, we found that Aplidin targets pre-osteoclasts and mature osteoclasts and potently inhibits their differentiation and bone resorptive activity. Further, Aplidin acts additively with Btz to inhibit MM cell-induced bone resorption in an ex vivo model of MM bone disease. The potent antiresorptive activity of Aplidin found in our studies, together with its potent activity against MM cells, strongly suggest
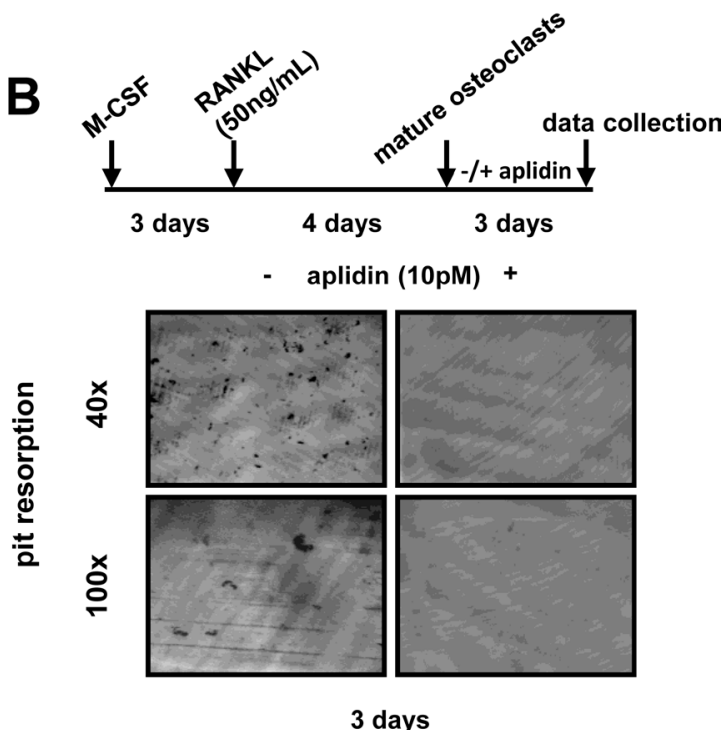

3 days

Figure 5: Aplidin decreases the number and activity of mature osteoclasts. (A) Number of TRAP + mature osteoclasts and protein expression of Cathepsin K after treatment with Aplidin for 3 days in the presence/absence of RANKL. (B) Resorption pits formed on bovine bone slices by mature osteoclasts treated with Aplidin for 3 days. Representative images out of 4 independent replicas are shown per each condition. Representative experiments out of two are shown ( $n=4$ per condition). Bars represent means $\pm \mathrm{SD}$. ${ }^{*} p<0.05$ Aplidin alone vs vehicle. 
that treatment with Aplidin could simultaneously block MM growth and bone destruction in MM patients.

Our studies show for the first time that Aplidin exhibits a potent anti-resorptive activity through direct actions on osteoclast precursors and mature osteoclasts. These cells exhibited a very high sensitivity to Aplidin compared to MM cells and other bone cells (picomolar versus nanomolar doses). Aplidin decreased Cathepsin K protein expression in mature osteoclasts, and prevented osteoclast differentiation by blocking the induction of osteoclast differentiation markers (NFATc1, TRAP, MMP-
9) and inhibiting the rapid ERK phosphorylation induced by RANKL signaling, key molecular events required for proper osteoclast formation and function [17]. This latter observation is in line with previous findings showing that Aplidin decreases ERK1/2 and ERK 5 phosphorylation in MM cells [9]. However, unlike in MM cells, Aplidin did not stimulate or altered RANKL-induced p38 phosphorylation, suggesting that Aplidin differentially regulates MAPK signaling pathways in non-tumor vs tumor cells.

High dose of glucocorticoids (GCs) is a frequent component of anti-MM therapy due to its ability to
A ex vivo bone organ culture
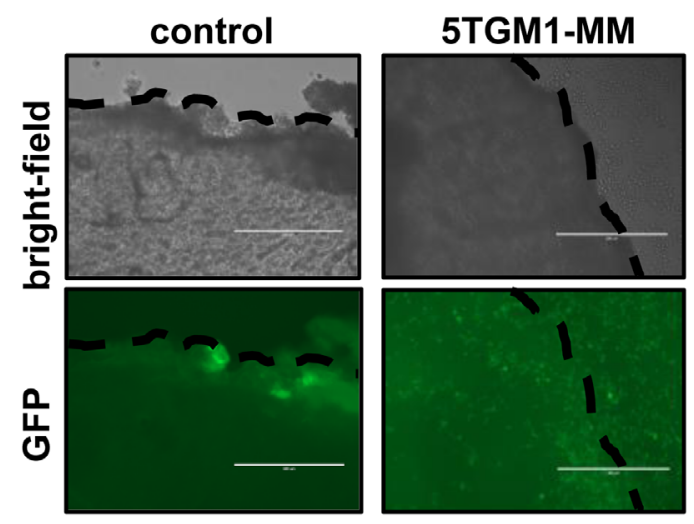

B

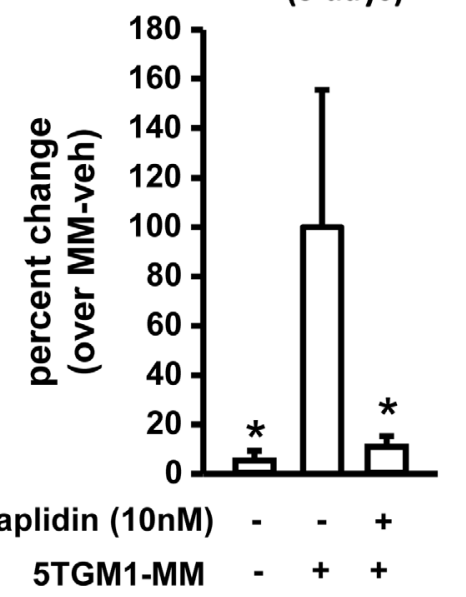

bone resorption (CTX) (3 days)

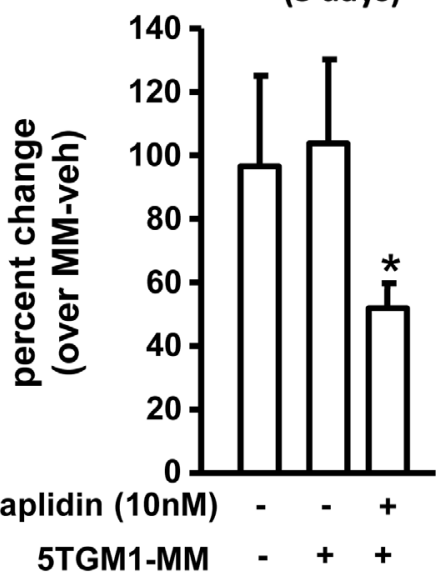

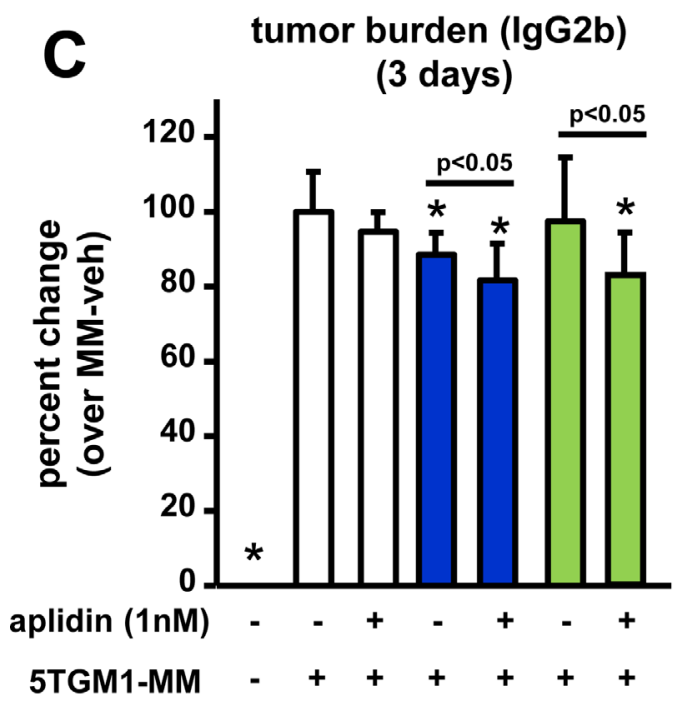

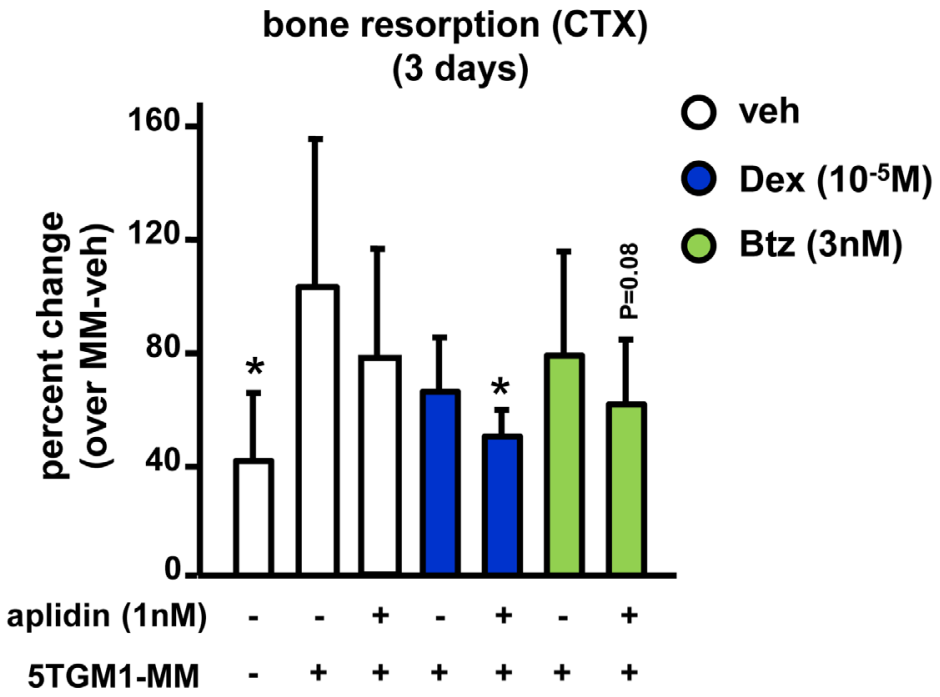

Figure 6: Aplidin decreases tumor growth and inhibits bone resorption in an ex vivo bone organ model of multiple myeloma. The levels of the tumor growth marker IgG2b and the bone resorption marker CTX were evaluated in conditioned media collected from control bones and bones bearing 5TGM1 MM cells. (A) Bright field pictures show the piece of calvarial bone and GFP fluorescent images show 5TGM1-GFP MM cells within the bone. Bones bearing MM cells were cultured with/without Aplidin alone (B) or combined with Dex and Btz (C). IgG2b and CTX levels were determined after 3 days. Percent change was calculated over bones bearing MM cells and cultured under vehicle conditions. Combined results of two independent ex vivo studies are shown. 4-6 independent calvarial bones were used per condition/experiment. Bars represent means $\pm \mathrm{SD} .{ }^{*} p<0.05$ vs MM-veh; lines indicate $p$ values $<0.05$ comparing MM-Dex/Btz alone vs MM-Dex/Btz + Aplidin. 
induce apoptosis of MM cells and capacity to enhance the efficacy of other chemotherapy drugs [18-20]. However, GCs cause rapid bone loss by increasing bone resorption, inducing osteoblast and osteocyte apoptosis, and reducing bone formation [21-23]. In contrast, Btz, also used as a frontline agent for the treatment of MM, can induce transient decreases in bone resorption and increase osteoblast number and bone formation markers [24]. Our in vitro results show that Aplidin counteracted the increase in osteoclasts differentiation induced by Dex, whereas in combination with Btz, it induced a more potent suppression of osteoclast differentiation and bone resorption than each agent alone. Together, these results show the potential of combining Aplidin with GCs or Btz for the treatment of MM, as these combination therapies could prevent tumor growth whilst reducing the bone loss induced by MM cells and mitigate the adverse effects of GCs on the skeleton.

Our proliferation and cell death studies in human and murine MM cells lines demonstrate that Aplidin exhibits anti-proliferative and pro-apoptotic activities in MM cells. In addition, combination of Aplidin with other anti-MM drugs induces better responses than each treatment alone, demonstrating that Aplidin enhances the anti-MM effects of Dex. This is consistent with previous findings showing that co-administration of Aplidin and Dex results in additive anti-MM effects [9], and with more recent results of phase III clinical trials demonstrating that patients with relapsed/refractory MM treated with combined combination of Aplidin and Dex had a significantly $35 \%$ lower risk of disease progression or death when compared to Dex alone (6). Further, Aplidin decreased the viability of JJN3 cells and enhanced their response to Btz. JJN3 cells have mutated p53, which can contribute to resistance to Btz and poor outcomes in MM patients [25]. Our findings are in line with a previous publication showing additive effects of Aplidin against MM cells when combined with Btz [9]. However, it remains unclear whether co-administration of Aplidin could overcome resistance to proteasome inhibitors and Dex. Together, these data suggest that Aplidin has a unique mechanism of action that does not interfere with pathways targeted by other anti-tumor drugs. In addition, our findings suggest that Aplidin could be effective against MM cells resistant to frequent components of anti-MM therapy and may help to overcome the drug resistance that patients develop over the course of treatment.

Treatment with Aplidin also induced cell death in osteocytes (via apoptosis), and osteoblasts, and enhanced the cell death induced by Dex in these cell types. Our results also suggest that co-administration of Aplidin and Dex leads to further decreases in tumor growth than each agent alone. However, this combination could further suppress osteoblast number in bones bearing MM. In contrast, we found that Btz alone did not affect osteoblast/ osteocyte viability, but it partially prevented the increased osteocyte/osteoblast cell death induced by Aplidin. This is consistent with a previous report showing that Btz prevents osteocyte cell death induced by Dex and MM cells in vitro, and that patients treated with Btz exhibit higher numbers of viable osteocytes compared with those not treated with Btz [26]. Mechanistic studies revealed that these protective effects of Btz are due to inhibition of osteocyte apoptosis and autophagic cell death [26]. Similar to a previous report

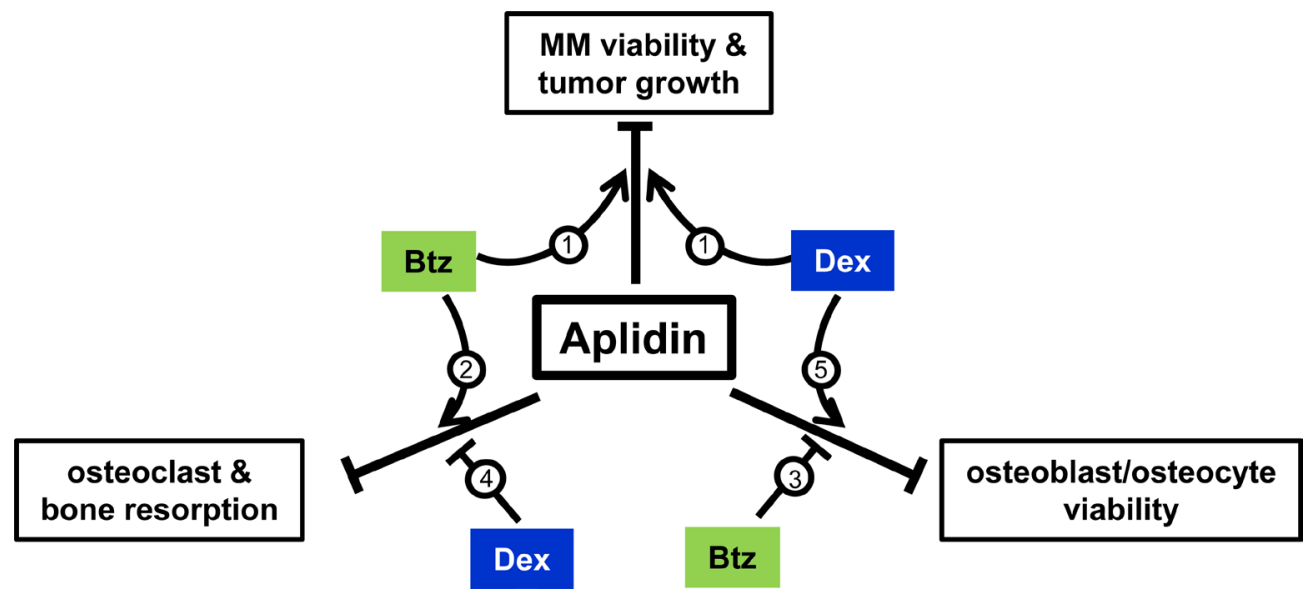

Figure 7: Working model. Aplidin inhibits MM cell viability, decreases osteoblast/osteocyte viability, and inhibits osteoclast differentiation and function. Co-administration of Aplidin enhances the anti-MM activity of Btz and Dex (1). Further, when combined with Aplidin, Btz increases the anti-resorptive properties of Aplidin (2), and partially prevents the decreases in osteoblast/osteocyte viability and osteoblast function induced by Aplidin (3). In contrast, Dex increases bone resorption (4) and in combination with Aplidin further decreases osteoblasts/osteocyte viability (5). Thus, administration of Aplidin as a single agent has the potential to simultaneously inhibit tumor growth and prevent bone destruction by inhibiting bone resorption. Moreover, in combination with Btz, it could result in superior anti-MM and anti-resorptive activities than each agent alone, as well as ameliorate the deleterious effects of Aplidin on osteoblasts/ osteocytes. Finally, although co-administration of Dex and Aplidin could also result in enhanced anti-MM effects, Dex may antagonize the anti-resorptive activity of Aplidin and further increase osteoblasts/osteocyte cell death. 
showing that Btz induced transient increases in osteoblast and circulating markers of bone formation [24], we found that Btz mitigated the reduction in osteoblast mineralization induced by Aplidin. Thus, combined administration of Aplidin and Btz could result in superior anti-MM activity, stronger suppression of bone resorption, and ameliorate the potential negative effects of Aplidin on bone formation. Future studies are warranted to examine the effects of Aplidin alone and in combination with current anti-MM drugs in in vivo models of MM-induced bone disease.

Our bone organ culture that reproduces the 3D-organization and the cellular diversity of the MM/ bone marrow niche allowed us to examine the effect of Aplidin simultaneously on MM and bone cells, thereby providing a model closer to an in vivo scenario. Using this innovative approach, we demonstrated that Aplidin decreased MM cell growth and osteoclast-mediated bone resorption in bones bearing $\mathrm{MM}$ cells, and that suboptimal doses of Aplidin in combination with Dex and Btz have additive effects against MM cells and decrease resorption to a larger extent that each treatment alone. These ex vivo results demonstrate that Aplidin can inhibit bone resorption in the $\mathrm{MM} /$ bone marrow microenvironment and provide additional support for combining Aplidin with other anti-MM drugs for the treatment of MM. However, further studies are needed to evaluate if Aplidin also inhibits bone resorption and has combinatorial effects when co-administered with other anti-MM drugs in murine models of MM disease and MM patients.

In summary, our study increases the potential of Aplidin as a therapy for MM by demonstrating its effects on MM-induced bone disease. We show that Aplidin is a potent anti-resorptive agent that inhibits bone resorption by acting directly on osteoclast precursors and mature osteoclasts. Further, the anti-tumor and anti-resorptive properties of Aplidin are preserved and/or enhanced when combined with frequent components of anti-MM therapy. Together, these findings provide the framework for additional preclinical and clinical studies of this agent for the treatment of MM and its associated skeletal disease.

\section{MATERIALS AND METHODS}

\section{Reagents}

RPMI-1640, $\alpha$-MEM, glutamine, penicillin, and streptomycin were purchased from Invitrogen Life Technologies (Grand Island, NY, USA). Aplidin was provided by PharmaMar S.A. (Madrid, Spain). Bortezomib (Btz) was purchased from Selleckchem.com (Houston, TX, USA), and Dexamethasone (Dex) from Sigma-Aldrich (St Louis, MO, USA). MTT from Invitrogen. Murine anti-p-ERK antibody (Cat. \# 9101), anti-ERK antibody (Cat. \#9102), anti-p-P38 antibody (Cat.\# 9211) and anti-P38 antibody (Cat.\#9212) and anti-GAPDH antibody (Cat.\#2118) were purchased from Cell Signaling Technology
(Danvers, MA, USA); $\beta$-ACTIN antibody (ab49900) from Abcam (Cambridge, MA, USA); anti-NFACT1 antibody (Cat.\# sc-7294); anti-MMP9 (Cat.\# sc-393859); anti-TRAP (Cat.\# sc-28204); and anti-Cathepsin K antibody (Cat.\# sc6507) from Santa Cruz Biotechnology Inc. (Dallas, TX, USA); and anti-DC-STAMP antibody (Cat.\# MABF39-1) from Millipore (Temecula, CA, USA). Human RANKL and M-CSF were purchased from R\&D systems (Minneapolis, MN, USA); and bone slices for osteoclast resorption from Immunodiagnostic Systems Inc. (Gaithersburg, MD, USA).

\section{Cells and culture conditions}

Human JJN3 and murine 5TGM1 MM cell lines, commonly used in our laboratory to model MM growth and bone disease in rodents [14, 30], were provided by N. Giuliani (University of Parma, Italy) and B. Oyajobi (University of Texas at San Antonio, TX, USA), respectively. Murine MLO-A5 osteocyte-like cells were provided by L. Bonewald (Indiana University, IN, USA) $[27,28]$. OB-6 osteoblast-like cells were obtained from R. Jilka (University of Arkansas for Medical Sciences) [29]. MC3T3-subclone MC4 pre-osteoblastic cells were obtained from G.D. Roodman (Indiana University). 5TGM1 and JJN3 MM cells, MC4 pre-osteoblastic cells, OB-6 osteoblastic cells, and MLO-A5 osteocyte-like cells were cultured as previously published [13, 14, 30]. All cell lines were tested for morphology, gene expression profile, and tumorigenic capacity (MM cells) according to previous publications and proved to be mycoplasma free.

\section{Cell viability}

5TGM1 and JJN3 MM cells $\left(3 \times 10^{5}\right.$ cells $\left./ \mathrm{mL}\right)$, and OB-6 osteoblastic cells and MLO-A5 osteocyte-like cells $\left(1.5 \times 10^{4} \mathrm{cell} / \mathrm{cm}^{2}\right)$ were treated for $24 \mathrm{~h}$ and 48 hours with Aplidin (1-180 nM) in the presence or absence of Btz $(3 \mathrm{nM})$ or Dex $\left(10^{-5} \mathrm{M}\right)$. Btz/Dex doses were selected based on previous studies showing that treatment with these concentrations decreases JJN3 and 5TGM1 cell viability by $\sim 50 \%$ [30], thus allowing us the detection of combinatorial effects when co-administered with Aplidin. JJN3 MM cells and MLO-A5 osteocyte-like cells were pre-incubated with DEVD (100 $\mathrm{nM})$ for $4 \mathrm{~h}$ and then treated with Aplidin 10nM for 48h. DEVD was refreshed every $24 \mathrm{~h}$. DMSO and ethanol were used as controls for these experiments. After treatment, 5TGM1 and JJN3 MM cells, OB-6 osteoblastic cells, and MLO-A5 osteocytelike cells were pelleted and resuspended in media. Cell death was quantified by Trypan blue uptake as described $[14,30]$, and cell viability was examined by MTT assays following protocols established by the manufacturer.

\section{Osteoblast differentiation}

MC-4 and OB-6 osteoblastic cells (5000 cells $\left./ \mathrm{cm}^{2}\right)$ were cultured with osteogenic media $(0.2 \mathrm{mM}$ ascorbic 
acid, $10 \mathrm{mM} \beta$-glycerophosphate) as previously described $[13,14]$, and treated with Aplidin (1 and $10 \mathrm{nM}$ ), alone or in combination with Btz $(3 \mathrm{nM})$ every 3 days or once per week. Media were replaced every 2-3 days, and mineralization was determined by Alizarin Red staining and quantified as described before [13].

\section{CFU-GM colony formation}

Murine non-adherent bone marrow cells $(1 \times$ $10^{5}$ cells/culture) were cultured in $\alpha$-MEM (Gibco, Grand Island, NY) containing 1.2\% methylcellulose, 30\% FBS, $1 \%$ deionized bovine serum albumin (BSA) (SigmaAldrich), and $100 \mathrm{ng} / \mathrm{mL}$ recombinant human GM-CSF (Immunex Corp., Seattle, WA, USA). The cells were plated in a volume of $1.0 \mathrm{~mL}$ in $35-\mathrm{mm}$ culture dishes (Corning, New York, NY), as reported previously [31], and treated with or without Aplidin (5 pM and $50 \mathrm{pM}$ ). The number of colonies that formed were scored after 7 days of culture using an inverted microscope.

\section{Osteoclast formation from murine bone marrow}

Bone marrow cells were flushed out from long bones of $\mathrm{C} 57 \mathrm{BL} / 6$ mice and cultured in $\alpha$-MEM containing $10 \%$ FBS overnight. Non-adherent cells were

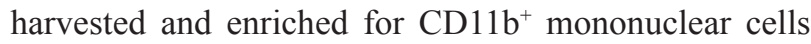
as described previously [32]. To determine the effects of Aplidin on the growth of osteoclast precursors, CD11 $\mathrm{b}^{+}$ cells were cultured in $\alpha$-MEM containing $10 \%$ FBS plus $10 \mathrm{ng} / \mathrm{mL}$ of M-CSF for 3 days in the presence or absence of Aplidin (0.01-10 pM). To assess the effects of Aplidin on osteoclast differentiation, $\mathrm{CD}_{11} \mathrm{~b}^{+}$cells were cultured in $\alpha$-MEM containing 10\% FBS plus $10 \mathrm{ng} / \mathrm{mL}$ of M-CSF for 3 days and then treated with RANKL (50 $\mathrm{ng} / \mathrm{mL})$ for 4 days in the presence/absence of Aplidin (0.01-10pM), Btz (3 nM), and Dex $\left(10^{-7} \mathrm{M}\right)$. RANKL and other treatments were replenished every two days. Cells were stained for TRAP using a leukocyte acid phosphatase kit (Sigma-Aldrich), and TRAP-positive mononuclear cells and multinuclear cells ( $\geq 3$ nuclei/cell) were scored microscopically after M-CSF for 3 days or after RANKL for 4 days respectively, as described previously [32].

\section{Isolation of mature osteoclasts from mouse bone marrow cultures and bone resorption assays}

Bone marrow cells were flushed out from long bones of C57BL/6 mice and cultured in a $10-\mathrm{cm}$ dish $\left(2.5 \times 10^{7}\right.$ cells/dish) with M-CSF (10 ng/ml) for 3 days and RANKL (50 $\mathrm{ng} / \mathrm{ml}$ ) for 4 days as previously described [32]. Then, cells were incubated with trypsin EDTA (Corning) for 3 minutes to remove non-osteoclastic cells and enrich the concentration of mature osteoclasts in the cultures. Mature osteoclasts $\left(1 \times 10^{4} /\right.$ well in 96 -well plates $)$ were treated with or without Aplidin (0.1-10 pM) for 3 days in the presence/absence of RANKL $(50 \mathrm{ng} / \mathrm{ml})$. For bone resorption assays, $5 \times 10^{4}$ mature osteoclasts were transferred to bovine slices and cultured with RANKL $(50 \mathrm{ng} / \mathrm{mL})$ and treated with Aplidin $(0.1$ and $10 \mathrm{pM})$ in the presence or absence of Btz $(3 \mathrm{nM})$ and Dex $\left(10^{-7} \mathrm{M}\right)$ for 3 or 7 days. RANKL $(50 \mathrm{ng} / \mathrm{mL})$ was added every two days to the resorption assays.

\section{Western blot analysis}

Protein lysates were prepared from osteoclast precursors undergoing differentiation and from mature osteoclasts as previously described [15]. Cell lysates (25 $\mu \mathrm{g})$ were boiled in the presence of sodium dodecyl sulfate (SDS) sample buffer (0.5 M Tris-HCl, pH 6.8, 10\% wt/vol SDS, $10 \%$ glycerol, $0.05 \% \mathrm{wt} / \mathrm{vol}$ bromophenol blue) for 5 minutes and subjected to electrophoresis on $4 \%$ to $20 \%$ SDS -PAGE (Bio-Rad Laboratories, Hercules, CA, USA). Proteins were transferred to nitrocellulose membranes using a semidry blotter (Bio-Rad) and incubated in blocking solution ( $5 \%$ nonfat dry milk in TBS containing $0.1 \%$ Tween-20) for 1 hour to reduce nonspecific binding. Membranes were washed extensively and immunoblots were performed using anti-p-ERK (1:1000), anti-ERK (1:1000), anti-p-P38 (1:1000), anti-P38 (1:1000), antiNFACT1 (1:1000), anti-CATHEPSIN K (1:1000), anti-DC-STAMP (1:500), anti-GAPDH (1:2000), antiMMP9 (1:1000), anti-TRAP ( $1: 200)$, and anti- $\beta$-ACTIN (1:4000) antibodies followed by rabbit anti-goat or goat anti-mouse antibodies respectively, conjugated to horseradish peroxidase (1:2000) in 5\% milk, Santa Cruz Biotechnology). Western blots were developed using an enhanced chemiluminescence detection assay following the manufacturer's directions (Bio-Rad).

\section{Ex vivo bone organ cultures}

Bone organ cultures were established as previously described [14]. Briefly, calvarial bones from 9-15 dayold mouse C57BL/6 pups were cut into $3.5 \mathrm{~mm}$ disks with a biopsy punch and placed into $24-w e l l$ plates. 5 $\times 10^{4} 5$ TGM1 MM cells were added in $0.5 \mathrm{ml}$ of RPMI medium supplemented with $10 \%$ fetal calf serum and antibiotics. Bones bearing MM cells were washed with PBS, transferred to a new plate, and treated with Aplidin $(1 \mathrm{nM}$ or $10 \mathrm{nM})$ in the presence or absence of Btz $(3 \mathrm{nM})$ and Dex $\left(10^{-5} \mathrm{M}\right)$. Conditioned media was collected 3 and 12 days after treatments for assessment of tumor growth by IgG2b levels and bone resorption markers by C-terminal telopeptides of type I collagen (CTX). Half of the media was replaced every 3 days until day 7 , when $100 \%$ of media was replaced with fresh culture media. Murine IgG2b concentrations in conditioned media were determined using commercially available enzyme-linked immunosorbent assays (ELISA) kits, according to manufacturer instructions (Bethyl Laboratories, Inc., Montgomery, TX, USA). CTX 
levels in conditioned media were quantified by ELISA (Immunodiagnostics Systems) as previously published [33].

\section{Statistics}

Data were analyzed using SigmaPlot 12.0 (Systat Software, Inc., San Jose, CA, USA). All values are reported as means $\pm \mathrm{SD}$. Differences between treatment groups were evaluated using One-way ANOVA. $p \leq 0.05$ was considered statistically significant.

\section{Study approval}

Studies using animal samples (calvarial bones) were approved by the Indiana University School of Medicine's IACUC.

\section{Author contributions}

J.D.C. and T.B. designed research; J.D.C., N.K., E.G.A., and J.H.N. performed research; J.D.C., G.D.R., C.M.G., and T.B. analyzed and interpreted data; J.D.C. and T.B. wrote the manuscript. All authors reviewed the manuscript.

\section{ACKNOWLEDGMENTS}

This work was supported by a restricted grant from PharmaMar S.A. (Madrid, Spain to T.B. and J.D.C), the National Institutes of Health (R01-CA209882 to G.D.R. and T.B.), the Veteran's Administration (Merit Reviews grant to T.B. and to G.D.R.), a Scholar Award by the American Society of Hematology Scholar Award (to J.D.C), and a Brian D. Novis Award by the International Myeloma Foundation (to J.D.C.).

\section{CONFLICTS OF INTEREST}

C.M.G. is an employee and a shareholder of PharmaMar S.A. G.D.R. has received consulting honoraria from Amgen Inc. The remaining authors declare no competing financial interests.

\section{REFERENCES}

1. Terpos E, Berenson J, Raje N, Roodman GD. Management of bone disease in multiple myeloma. Expert Rev Hematol. 2014; 7:113-25. https://doi.org/10.1586/17474086.2013.87 4943. [PubMed]

2. Bianchi G, Munshi NC. Pathogenesis beyond the cancer clone(s) in multiple myeloma. Blood. 2015; 125:3049-58. https://doi.org/10.1182/blood-2014-11-568881. [ubMed]

3. Croucher PI, McDonald MM, Martin TJ. Bone metastasis: the importance of the neighbourhood. Nat Rev Cancer. 2016; 16:373-86. https://doi.org/10.1038/nrc.2016.44. [PubMed]
4. Silbermann R, Roodman GD. Myeloma bone disease: Pathophysiology and management. J Bone Oncol. 2013; 2:59-69. https://doi.org/10.1016/j.jbo.2013.04.001. [PubMed]

5. Silbermann R, Roodman GD. Bone effects of cancer therapies: pros and cons. Curr Opin Support Palliat Care. 2011; 5:251-7. https://doi.org/10.1097/ SPC.0b013e328349c524. [PubMed]

6. Alonso-Alvarez S, Pardal E, Sanchez-Nieto D, Navarro M, Caballero MD, Mateos MV, Martin A. Plitidepsin: design, development, and potential place in therapy. Drug Des Devel Ther. 2017; 11:253-64. https://doi.org/10.2147/ DDDT.S94165. [PubMed]

7. Losada A, Munoz-Alonso MJ, Garcia C, Sanchez-Murcia PA, Martinez-Leal JF, Dominguez JM, Lillo MP, Gago F, Galmarini CM. Translation Elongation Factor eEF1A2 is a Novel Anticancer Target for the Marine Natural Product Plitidepsin. Sci Rep. 2016; 6:35100. https://doi.org/10.1038/ srep35100. [PubMed]

8. Munoz-Alonso MJ, Gonzalez-Santiago L, Martinez T, Losada A, Galmarini CM, Munoz A. The mechanism of action of plitidepsin. Curr Opin Investig Drugs. 2009; 10:536-42. [PubMed]

9. Mitsiades CS, Ocio EM, Pandiella A, Maiso P, Gajate C, Garayoa M, Vilanova D, Montero JC, Mitsiades N, McMullan CJ, Munshi NC, Hideshima T, Chauhan D, et al. Aplidin, a marine organism-derived compound with potent antimyeloma activity in vitro and in vivo. Cancer Res. 2008; 68:5216-25. https://doi.org/10.1158/0008-5472.CAN-075725. [PubMed]

10. Caers J, Menu E, De Raeve H, Lepage D, Van Valckenborgh E, Van Camp B, Alvarez E, Vanderkerken K. Antitumour and antiangiogenic effects of Aplidin in the 5TMM syngeneic models of multiple myeloma. $\mathrm{Br} \mathrm{J}$ Cancer. 2008; 98:1966-74. https://doi.org/10.1038/sj.bjc.6604388. [PubMed]

11. Spicka I, Ocio EM, Oakervee HE, Greil R, Banh RH, Catley L, Huang S, D'Rozario JM, Dimopoulos MA, Martinez S, Extremera S, Alfaro V, Carella AM, et al. Randomized Phase III Study (ADMYRE) of Plitidepsin in Combination with Dexamethasone Vs. Dexamethasone Alone in Patients with Relapsed/Refractory Multiple Myeloma. Blood. 2017; 130:1886. https://doi.org/10.1200/ jco.2018.36.15 suppl.8018.

12. Sato AY, Cregor M, Delgado-Calle J, Condon KW, Allen MR, Peacock M, Plotkin LI, Bellido T. Protection from glucocorticoid-induced osteoporosis by anti-catabolic signaling in the absence of Sost/sclerostin. J Bone Miner Res. 2016; 31:1791-802. https://doi.org/10.1002/jbmr.2869. [PubMed]

13. Delgado-Calle J, Hancock B, Likine EF, Sato AY, McAndrews K, Sanudo C, Bruzzaniti A, Riancho JA, Tonra JR, Bellido T. MMP14 is a novel target of PTH signaling in osteocytes that controls resorption by regulating soluble RANKL production. FASEB J. 2018; 32:2878-2890. https://doi.org/10.1096/fj.201700919RRR. [PubMed] 
14. Delgado-Calle J, Anderson J, Cregor MD, Hiasa M, Chirgwin JM, Carlesso N, Yoneda T, Mohammad KS, Plotkin LI, Roodman GD, Bellido T. Bidirectional Notch signaling and osteocyte-derived factors in the bone marrow microenvironment promote tumor cell proliferation and bone destruction in multiple myeloma. Cancer Res. 2016; 76:1089-100. https://doi.org/10.1158/0008-5472.CAN-151703. [PubMed]

15. Tu X, Delgado-Calle J, Condon KW, Maycas M, Zhang H, Carlesso N, Taketo MM, Burr DB, Plotkin LI, Bellido T. Osteocytes mediate the anabolic actions of canonical Wnt/bcatenin signaling in bone. Proc Natl Acad Sci U S A. 2015; 112:E478-E486. https://doi.org/10.1073/pnas.1409857112. [PubMed]

16. Ben-awadh AN, Delgado-Calle J, Tu X, Kuhlenschmidt K, Allen MR, Plotkin LI, Bellido T. Parathyroid hormone receptor signaling induces bone resorption in the adult skeleton by directly regulating the RANKL gene in osteocytes. Endocrinology. 2014; 155:2797-809. https:// doi.org/10.1210/en.2014-1046. [PubMed]

17. Nakamura H, Hirata A, Tsuji T, Yamamoto T. Role of osteoclast extracellular signal-regulated kinase (ERK) in cell survival and maintenance of cell polarity. J Bone Miner Res. 2003; 18:1198-205. https://doi.org/10.1359/ jbmr.2003.18.7.1198. [PubMed]

18. Feinman R, Koury J, Thames M, Barlogie B, Epstein J, Siegel DS. Role of NF-kappaB in the rescue of multiple myeloma cells from glucocorticoid-induced apoptosis by bcl-2. Blood. 1999; 93:3044-52. [PubMed]

19. Chauhan D, Hideshima T, Pandey P, Treon S, Teoh G, Raje N, Rosen S, Krett N, Husson H, Avraham S, Kharbanda S, Anderson KC. RAFTK/PYK2-dependent and -independent apoptosis in multiple myeloma cells. Oncogene. 1999; 18:6733-40. https://doi.org/10.1038/sj.onc.1203082. [PubMed]

20. Chauhan D, Pandey P, Ogata A, Teoh G, Treon S, Urashima M, Kharbanda S, Anderson KC. Dexamethasone induces apoptosis of multiple myeloma cells in a JNK/SAP kinase independent mechanism. Oncogene. 1997; 15:837-43. https://doi.org/10.1038/sj.onc.1201253. [PubMed]

21. Sato AY, Peacock M, Bellido T. Glucocorticoid excess in bone and muscle. Clin Rev Bone Miner Metab. 2018; 16:33-47. https://doi.org/10.1007/s12018-018-9242-3. [PubMed]

22. Weinstein RS. Glucocorticoid-induced osteoporosis and osteonecrosis. Endocrinol Metab Clin North Am. 2012; 41:595-611. https://doi.org/10.1016/j.ecl.2012.04.004. [PubMed]

23. Weinstein RS. Clinical practice. Glucocorticoid-induced bone disease. N Engl J Med. 2011; 365:62-70. https://doi. org/10.1056/NEJMcp1012926. [PubMed]

24. Accardi F, Toscani D, Costa F, Aversa F, Giuliani N. The Proteasome and Myeloma-Associated Bone Disease. Calcif Tissue Int. 2018; 102:210-26. https://doi.org/10.1007/ s00223-017-0349-1. [PubMed]
25. Ling X, Calinski D, Chanan-Khan AA, Zhou M, Li F. Cancer cell sensitivity to bortezomib is associated with survivin expression and p53 status but not cancer cell types. J Exp Clin Cancer Res. 2010; 29:8. https://doi. org/10.1186/1756-9966-29-8. [PubMed]

26. Toscani D, Palumbo C, Dalla Palma B, Ferretti M, Bolzoni M, Marchica V, Sena P, Martella E, Mancini C, Ferri V, Costa F, Accardi F, Craviotto L, et al. The Proteasome Inhibitor Bortezomib Maintains Osteocyte Viability in Multiple Myeloma Patients by Reducing Both Apoptosis and Autophagy: A New Function for Proteasome Inhibitors. J Bone Miner Res. 2016; 31:815-27. https://doi. org/10.1002/jbmr.2741. [PubMed]

27. Kato Y, Boskey A, Spevak L, Dallas M, Hori M, Bonewald LF. Establishment of an osteoid preosteocyte-like cell MLO-A5 that spontaneously mineralizes in culture. J Bone Miner Res. 2001; 16:1622-33. https://doi.org/10.1359/ jbmr.2001.16.9.1622. [PubMed]

28. Kato Y, Windle JJ, Koop BA, Mundy GR, Bonewald LF. Establishment of an osteocyte-like cell line, MLOY4. J Bone Miner Res. 1997; 12:2014-23. https://doi. org/10.1359/jbmr.1997.12.12.2014. [PubMed]

29. Lecka-Czernik B, Gubrij I, Moerman EJ, Kajkenova O, Lipschitz DA, Manolagas SC, Jilka RL. Inhibition of Osf $2 / \mathrm{Cbfa} 1$ expression and terminal osteoblast differentiation by PPAR-gamma 2. J Cell Biochem. 1999; 74:357-71. https://doi.org/10.1002/(SICI)10974644(19990901)74:3<357::AID-JCB5>3.0.CO;2-7. [PubMed]

30. Delgado-Calle J, Anderson J, Cregor MD, Condon KW, Kuhstoss SA, Plotkin LI, Bellido T, Roodman GD. Genetic deletion of sost or pharmacological inhibition of sclerostin prevent multiple myeloma-induced bone disease without affecting tumor growth. Leukemia. 2017; 31:2686-94. https://doi.org/10.1038/leu.2017.152. [PubMed]

31. Teramachi J, Silbermann R, Yang P, Zhao W, Mohammad KS, Guo J, Anderson JL, Zhou D, Feng R, Myint KZ, Maertz N, Beumer JH, Eiseman JL, et al. Blocking the ZZ domain of sequestosome1/p62 suppresses myeloma growth and osteoclast formation in vitro and induces dramatic bone formation in myeloma-bearing bones in vivo. Leukemia. 2016; 30:390-8. https://doi.org/10.1038/leu.2015.229. [PubMed]

32. Teramachi J, Nagata Y, Mohammad K, Inagaki Y, Ohata Y, Guise T, Michou L, Brown JP, Windle JJ, Kurihara N, Roodman GD. Measles virus nucleocapsid protein increases osteoblast differentiation in Paget's disease. J Clin Invest. 2016; 126:1012-22. https://doi.org/10.1172/JCI82012. [PubMed]

33. Delgado-Calle J, Tu X, Pacheco-Costa R, McAndrews K, Edwards R, Pellegrini G, Kuhlenschmidt K, Olivos N, Robling A, Peacock M, Plotkin LI, Bellido T. Control of bone anabolism in response to mechanical loading and PTH by distinct mechanisms downstream of the PTH receptor. $\mathrm{J}$ Bone Miner Res. 2017; 32:522-35. https://doi.org/10.1002/ jbmr.3011. [PubMed] 